\title{
ORIGINAL RESEARCH \\ Pattern of Cortical Changes in Sporadic Creutzfeldt-Jakob Disease
}

\section{H.J. Tschampa}

K. Kallenberg

H.A. Kretzschmar

B. Meissner

M. Knauth

H. Urbach

I. Zerr
BACKGROUND AND PURPOSE: High cortical signal intensity on diffusion-weighted (DW) or fluid-attenuated inversion recovery (FLAIR) images is increasingly described in sporadic Creutzfeldt-Jakob disease (sCJD). The aim of this study was to assess the extent and location of high cortical signal intensity, to investigate whether DW or FLAIR is superior in showing changes in cortical signal intensity, and to find out whether the distribution of the signal intensity changes is random or follows a common pattern.

MATERIALS AND METHODS: We analyzed FLAIR and DW MR imaging scans of 39 patients with sCJD for hyperintense cortical signal intensity. We compared the sensitivity of the DW and FLAIR scans. We correlated the extent and location of the cortical signal intensity changes with concomitant changes in deep gray matter and the genotype of codon 129 of the prion protein gene.

RESULTS: There was high signal intensity in the insula, the cingulate gyrus, and the superior fronta gyrus in $95 \%$. The cortical areas near the midline also frequently showed the abnormal signal intensity (precuneus $87 \%$, paracentral lobe $77 \%$ ). The precentral and postcentral gyri were affected less frequently $(41 \%$ and $28 \%$, respectively). The DW MR imaging showed the cortical changes more effectively than FLAIR. There was no correlation between the distribution of changes and additional signal alterations in deep gray matter or the genotype of codon 129

CONCLUSION: The distribution of cortical signal intensity abnormalities in patients with SCJD follows a common pattern, affecting mainly the cortical areas near the midline, the insula, cingulum, and the superior frontal cortex. DW imaging is superior to FLAIR in the detection of cortical high signal intensity.
C reutzfeldt-Jakob disease (CJD) is a rare, fatal, neurodegenerative disorder characterized by rapidly progressive dementia and neurologic signs. CJD belongs to the group of transmissible spongiform encephalopathies, which share the neuropathologic triad of spongiform degeneration of the brain, neuronal death, and astrocytic gliosis. ${ }^{1}$ There are different forms of CJD. The most common form $(85 \%)$ is sporadic CJD (sCJD); the cause of the disease is unknown. The clinical diagnosis of SCJD is based on clinical signs, characteristic results on electroencephalography (EEG), and detection of the protein 14-3-3 in the CSF. ${ }^{1}$ Typical findings on MR imaging in sCJD are signal hyperintensity abnormalities in the cortical and deep gray matter. ${ }^{2-5}$ The first reports, relying mainly on T2- and proton attenuation-weighted sequences, focused on hyperintense and often symmetrical high signal intensity in the head of the caudate nucleus and the putamen. ${ }^{2,6,7}$ The sensitivity of striatal MR imaging changes in the diagnosis of

Received July 26, 2006; accepted after revision October 30 .

From the Department of Radiology (Neuroradiology) (H.J.T., H.U.), Friedrich-Wilhelms University of Bonn, Germany; Departments of Neurology (I.Z., B.M.) and Neuroradiology (K.K., M.K.), Georg-August University, Göttingen, Germany; and Department of Neuropathology (H.A.K.), Ludwig-Maximilians University, Munich, Germany.

H.U. and I.Z. contributed equally to this work

This study was supported by grants from the Bundesministerium für Gesundheit und Soziales (BMGS), grant Az 325-4471-02/15 (to H.A.K.); the Bundesministerium für Bildung und Forschung (BMBF), grant D1GI0301 (to I.Z.); the European Commission, grant QLG3CT-2002-81606 (to I.Z.).

Paper previously presented at: Annual Meeting of the Radiological Society of North America, December 1, 2005; Chicago, III.

Address correspondence to Henriette Tschampa, Department of Radiology, Neuroradiology, University of Bonn, Sigmund-Freud-Str 25, 53105 Bonn, Germany; e-mail: henriette.tschampa@ukb.uni-bonn.de

Indicates article with supplemental on-line tables

DOI 10.3174/ajnr.A0496
sCJD is reported in $79 \%{ }^{6}$ and $39 \%{ }^{7}$ of patients. With the introduction of fluid-attenuated inversion recovery (FLAIR) ${ }^{8}$ and diffusion-weighted (DW $)^{4,9,10}$ imaging, cortical ribbonlike high-signal intensity has increasingly been described. ${ }^{11}$ The inclusion of abnormal cortical signal intensity in the MR diagnosis of sCJD increases sensitivity, specificity, and accuracy to $>91 \%{ }^{4}$ In 1999 , Parchi et al ${ }^{12}$ established the molecular basis for pathologically distinct phenotypes of sCJD. They proposed a classification of sCJD according to homozygosity or heterozygosity for methionine $(\mathrm{M})$ or valine $(\mathrm{V})$ at the polymorphic codon 129 of the prion protein gene, and the electrophoretic mobility of the protease-resistant fragment of the pathologic prion protein (types 1 or 2). Six subtypes were defined, designated MM1, MM2, MV1, MV2, VV1, and VV2. The molecular subtypes differ concerning age of onset, duration of the disease, EEG and 14-3-3 findings, and distribution of histopathologic changes. ${ }^{7,12,13}$ There are few data on MR imaging results among the molecular subtypes, suggesting that there might also be differences in the extent and distribution of signal hyperintensity changes. ${ }^{13-17}$

Other forms of CJD are variant CJD (vCJD), familial or genetic prion diseases, and iatrogenic CJD. Although familial or genetic CJD and iatrogenic forms are very rare, vCJD is frequent mainly in the United Kingdom and is probably associated with bovine spongiform encephalopathy. Patients with vCJD have high MR signal intensity in the pulvinar thalami on FLAIR, T2-, and proton attenuation-weighted images. This "pulvinar sign" (or "hockey-stick sign" if there is additional involvement of the medial thalamic nucleus) is considered pathognomonic for vCJD and is included in the World Health Organization diagnostic criteria. ${ }^{18}$

In the present study, we analyze the extent and distribution of signal intensity changes in a group of 39 patients with sCJD 
with cortical signal intensity abnormalities to find out whether the high signal intensity changes are distributed randomly or whether they share a common pattern. In addition, we investigate whether the distribution of the cortical signal intensity changes correlates with the involvement of the areas of deep gray matter and whether there are differences in the distribution of signal intensity changes among molecular sCJD subtypes. We compare FLAIR and DW images to determine which sequence is more effective in showing cortical signal intensity changes.

\section{Materials and Methods}

\section{Patients}

We studied MR imaging scans of 39 patients with sCJD: 20 were neuropathologically confirmed (definite sCJD), ${ }^{19}$ and 19 were classified as having probable sCJD. ${ }^{1}$ We did not include the cases of patients with possible sCJD. ${ }^{1}$ Patients were referred to the German CJD Surveillance Unit in the years 2001-2003, as described previously. ${ }^{3}$ In our study group, 19 patients were female, and 20 patients were male. The mean age at onset of disease was 64 years ( \pm 8.7 years). The mean time from onset of disease to MR imaging was 3 months ( \pm 2.5 months). The genotype at the polymorphic codon 129 of the prion protein gene was known in 34 of the 39 patients: 25 were homozygous for methionine (MM); 5, homozygous for valine (VV); and 4, heterozygous $(\mathrm{MV})$. The pathologic prion-protein type $\left(\mathrm{PrP}^{\mathrm{Sc}}\right)$ was determined in 11 patients: 8 had $\operatorname{PrP}^{S c}$ type 1 and 3 had $\operatorname{PrP}^{S c}$ type2. Combining codon 129 and $\mathrm{PrP}^{\mathrm{Sc}}$ type gave the following molecular subtypes ${ }^{12}: 7$ patients with MM1, 2 patients with MM2, and 1 patient each with MV2 or VV1.

\section{MR Imaging Analysis}

The study group was derived from a larger group of patients with CJD described previously in detail. ${ }^{3}$ As a prerequisite, only those patients with SCJD whose MR images showed signal hyperintensity in at least 1 cerebral cortical area and who had a whole-brain MR examination of adequate image quality, including FLAIR and/or diffusionweighted scans, were included in the present study. Eighteen patients had both FLAIR and DW scans, 13 patients had FLAIR, and 8 had DW sequences only. In total, 31 FLAIR and 26 DW scans from 39 patients were available. For analysis, we used the hard-copy documentations of axial scans. Because patients were referred from institutions all over Germany, they had been examined on different scanners: 30 patients on a $1.5 \mathrm{~T}$ scanner and 7 patients on a $1 \mathrm{~T}$ system. In 2 patients, the field strength was not given on the hard copies. Scan parameters differed little between the institutions; in most DW images, diffusion gradient strengths (b-values) of $1000 \mathrm{~s} / \mathrm{mm}^{2}$ were used.

Two observers (H.J.T., H.U.) analyzed the MRIs in consensus. The 2 radiologists recorded the cortical regions ${ }^{20}$ with abnormal signal intensity. Abnormal cortical signal intensity was defined as a ribbonlike hyperintensity in the cortex considerably different from other cortical areas of the same patient. We took into account the normal variability of MR signal intensity in the cerebral cortex with higher signal intensity in the insula, the cingulate gyrus, and the basal and medial temporal cortices in the studied sequences. ${ }^{21}$ Thus, the slight increases in signal intensity only in the aforementioned brain areas were not accepted as abnormal. We recalled DW and FLAIR images simultaneously and noted on which sequence the signal intensity changes were easier to detect. Abnormal cortical signal intensity was recorded for a gyrus if the signal intensity changes were detectable at

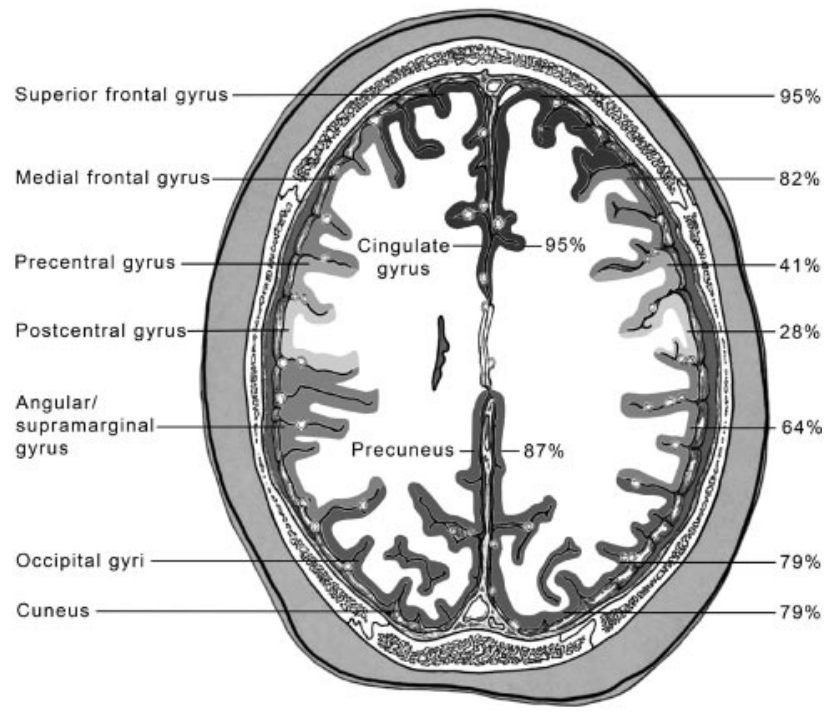

Fig 1. Modified section from a brain atlas ${ }^{20}$ showing the brain regions studied (right side) and the frequency (left side) in which cortical signal intensity abnormalities were detected. The gray-scale indicates the frequency in which the region shows hyperintensity, ranging from slight gray (postcentral gyrus, 28\%) to dark gray (insula and superior frontal and cingulate gyri, 95\%). [Reprinted with permission.]

least on 1 hemisphere. We noted whether the signal intensity abnormalities were symmetrical or whether they were predominant on 1 side.

We compared the distribution of the signal intensity changes among the different genotypes. For statistical analysis, the $\chi^{2}$ test corrected for multiple testing was used. Corrected $P$ values $<.05$ were considered statistically significant.

\section{Results}

\section{Distribution of Cortical Signal Intensity Changes}

There was widespread involvement of the cerebral cortex, which is summarized in Fig 1.

Most frequently, the insula, the cingulate, and the superior frontal gyri were affected in 37 of 39 patients (95\%). This was followed by the cortical areas near the midline, such as the precuneus in 34 of 39 patients (87\%), the cuneus in 31 of 37 patients $(79 \%)$, and the paracentral lobe in 30 of 39 patients $(77 \%)$. More than two thirds of the patients had high signal intensity in the medial frontal gyrus (32/39) and the occipital gyrus (31/39). In most patients, the angular/supramarginal gyrus (25/39 patients), and the superior parietal (24/39) and inferior frontal gyri (21/39) were affected. Abnormal signal intensity in the primary sensory cortex (postcentral gyrus, $11 / 39$ patients [28\%]), the primary motor cortex (precentral gyrus, $16 / 39$ patients $[41 \%])$, and the medial (15/39) and superior temporal gyri (12/39) was less common. Asymmetric lesions were present in 16 of 39 patients (41\%): in 10 patients (26\%), high signal intensity predominated on the left side and in 6 patients $(15 \%)$, on the right. In all patients, the hyperintense lesions were not completely confined to 1 hemisphere, but there were slight changes also on the contralateral side. In summarizing the signal intensity changes for the different lobes, the frontal lobe was involved in $100 \%$ of patients, the parietal lobe, the cingulate gyrus, and the insula showed abnormal signal intensity in $95 \%$. The occipital lobe was in- 
volved in $85 \%$ and the temporal lobe, to a lesser extent, in $49 \%$.

\section{Involvement of Deep Gray Matter}

The criterion for selection for inclusion into this study was the presence of cortical signal intensity changes. Among the 39 patients studied, 3 patients had cortical hyperintensity only; 16 patients had cortical changes and, additionally, high signal intensity in the striatum; and the remaining 20 patients had a combination of cortical, striatal, and thalamic signal intensity abnormalities. The distribution of cortical changes did not differ significantly among these 3 groups. The $\chi^{2}$ test corrected for multiple testing demonstrated $P>.05$ in all cortical regions (On-line Table 1).

\section{Comparison of MR Images}

Compared with FLAIR, it was easier to detect the hyperintense cortical signal intensity changes on the DW images. Sensitivity for the detection of signal intensity changes was $74 \%$ (23/31 patients) on the FLAIR images and 96\% (25/26 patients) on the DW images. Both sequences were available in 18 patients: on visual assessment, the DW images were judged to be more effective than FLAIR in 14 patients. Indeed, in 8 patients, the cortical lesions were discernible only on the DW images. In 6 patients, the abnormal signal intensity was more easily detected on the DW than on the FLAIR images. In 3 patients, both the FLAIR and DW images showed the signal intensity changes equally. In 1 patient, there was low image quality of the DW images that led to a better detection of signal intensity changes on the FLAIR images. There was no statistically significant difference between the FLAIR and DW sequences in the frequency of hyperintensity in the different cortical regions $\left(\chi^{2}\right.$ test, corrected for multiple testing: $P>.05$ in all cortical regions; On-line Table 2 ).

\section{Correlation with Molecular Subtypes}

According to the genotype at codon 129 of the prion protein gene, stratification of patients showed some differences in the distribution of the cortical signal intensity changes (eg, high signal intensity in the occipital cortex was present in $96 \%$ of patients with the MM genotype, but in only $60 \%$ of patients homozygous for valine and 50\% of heterozygous patients). These differences were not significant $\left(\chi^{2}\right.$ test corrected for multiple testing: $P>.05$ in all cortical regions, On-line Table 3).

\section{Discussion}

Since the introduction of FLAIR and DW MR images, signal hyperintensity changes in the cerebral cortex of patients with sCJD have increasingly been reported. ${ }^{4,5,8,11}$

To our knowledge, this is the first systematic study on the extent and distribution of cortical signal intensity changes in a large group of patients with sCJD. We found extensive cortical involvement, predominantly in the insula, the cingulate gyrus, the frontal gyri, and cortical areas situated near the midline. The central region including the precentral and postcentral gyri was involved to a lesser extent. Ninety-five percent of the studied patients had a combination of signal hyperintensity alterations in at least 3 of the 4 following brain areas: insula,
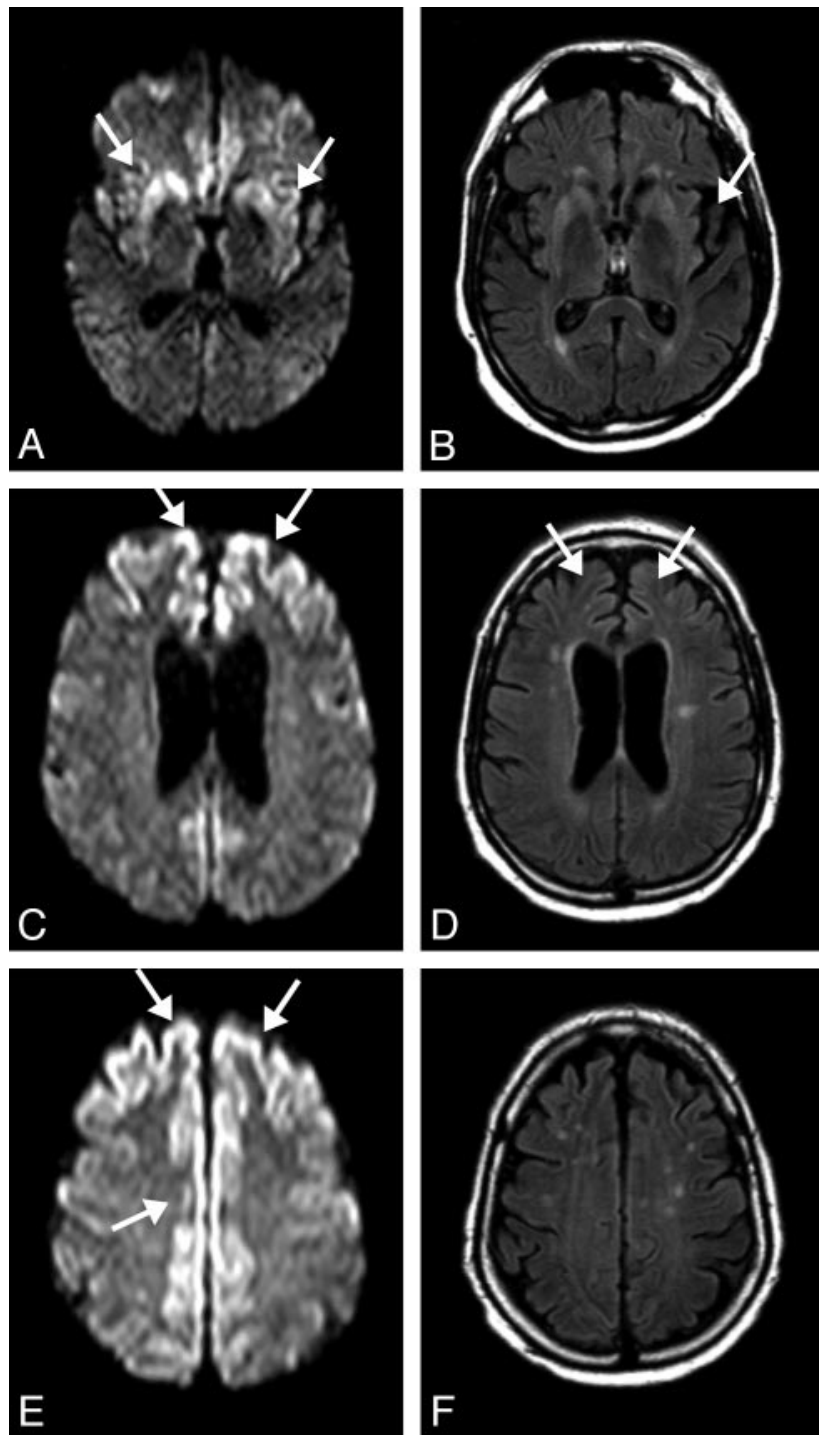

Fig 2. MR imaging scans of a 57-year-old patient with probable sporadic CJD 8 months after onset of disease. On DW MR imaging $(A, C, E)$, there is hyperintensity in the striatum and insula $(A)$, the superior and middle frontal gyri, and the precuneus $(C)$, the superior and middle frontal gyri, the precuneus, and the paracentral lobe $(E)$. On FLAIR $(B, D)$, the insula and cingulate gyrus show increased signal intensity, whereas the hyperintense changes in the precuneus and paracentral lobe, easily identified on DW imaging $(E)$, are questionable on FLAIR $(F)$.

cingulate gyrus, superior frontal gyrus, and occipital gyrus (Figs 1-3). Asymmetric findings with predominance of 1 side were present in $40 \%$ of patients. These results suggest a common pattern of cortical involvement in SCJD. Our findings are in accordance with those from Young et al, ${ }^{4}$ who reported an affection of up to $84 \%$ in the frontal cortex and a sparing of the rolandic area. ${ }^{22}$

\section{Comparison of FLAIR and DW Imaging}

We were able to confirm that DW images have a higher conspicuousness for the detection of cortical changes compared with FLAIR (Figs 2 and 3). ${ }^{4,9-11,23}$ Whether the high signal intensity was detected on DW images only or on DW images and FLAIR images, there was no statistically significant difference in the distribution of signal intensity changes (On-line Table 2). We thus conclude that DW and FLAIR images show 

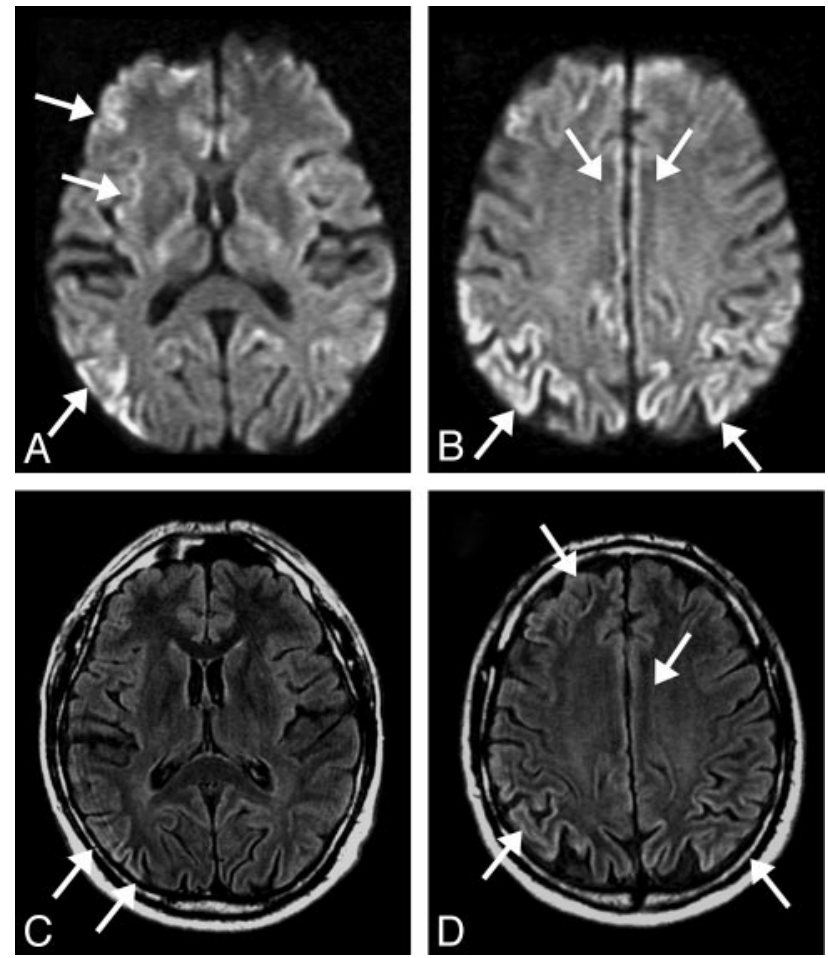

Fig 3. DW $(A, B)$ and FLAIR $(C, D)$ MR imaging of a 63-year-old patient with definite sporadic CJD after 4 months of disease. There is extensive signal hyperintensity, including the cingulate gyrus $(A-D)$, the frontal superior gyrus $(A-D)$, occipital gyrus $(A, C)$, the insula $(A, C)$, the temporal medial gyrus $(A, C)$, and the angular/supramarginal gyrus $(B, D)$. The basal ganglia have normal signal intensity $(A, C)$. The changes in signal intensity are easier to identify on $\mathrm{DW}(A, B)$ than on FLAIR imaging $(C, D)$. (Images courtesy of $\mathrm{H}$. Zeumer, Department of Neuroradiology, University Hospital Hamburg-Eppendorf, Germany.)

the same cortical signal intensity changes, but the use of DW imaging provides an increased probability to detect them. In our present study, we had hard-copy documentation of the DW scans (generally with b-values of $1000 \mathrm{~s} / \mathrm{mm}^{2}$ ). Young et $\mathrm{al}^{4}$ reported an easier detection of signal intensity changes by using soft-copy images. Rowley et $\mathrm{al}^{24}$ described a very high sensitivity of $b$-value $\left(b=2500 \mathrm{~s} / \mathrm{mm}^{2}\right)$ DW MR imaging in the diagnosis of CJD. Therefore, we cannot exclude the idea that the cortical signal intensity abnormalities in CJD will be even more widespread using higher b-value DW images or a digital display system that would allow the adjustment of window and level.

\section{Pathophysiology of Signal Intensity Changes and Molecular Subtypes}

The reason for cortical signal intensity changes in SCJD is not fully understood. Several studies have shown that the intensity of MR changes correlates with the neuropathologic findings (spongiosis, neuronal loss, and astrogliosis). 5,25-27 In areas that are bright on MR imaging in FLAIR or DW images, neuropathologic changes are more severe than in areas with normal cortical signal intensity. ${ }^{26,27}$ The hypothesis that MR signal intensity changes reflect pathologic conditions is additionally supported by clinicoradiologic comparison studies showing that cortical signal intensity changes correlate with clinical signs (eg, affection of the occipital cortex and visual disturbances $^{28}$ or unilateral cortical affection and contralateral motor signs). ${ }^{29}$
Concerning the cause of the distribution of lesions, we propose 2 hypotheses: 1) predominant manifestation of the disease in cortical areas with signal hyperintensity in MR imaging and 2) a sequence-inherent higher sensitivity in cortical areas bright on MR imaging as a result of intrinsic variations in the water content of different cortical regions in a diffuse cortical process.

We are not aware of a systematic study that would compare the distribution of signal intensity changes on MR imaging and patterns of neuropathologic lesions. To our knowledge, the largest study on neuropathologic lesion patterns in SCJD (not dealing with MR imaging) was published by Parchi et al in 1999. ${ }^{12}$ They showed that the subtypes of sCJD have differences in the severity of pathologic changes in different brain areas, including the basal ganglia and cortex. In the most frequent sCJD subtype in Western countries (MM1), the severest affection was in the occipital cortex, followed by the parietal, frontal, and temporal cortices. ${ }^{12}$ The insula and the cingulate gyrus were not listed separately in their article. Patients with the MM1 subtype had predominantly pathologic changes in the cortex and fewer pathologic changes in the basal ganglia, whereas patients with the second most common subtype (VV2) were described with more severe pathologic changes in the basal ganglia/thalamus than in the cortex. They reported that the frontal cortex was more severely affected than the occipital cortex in the patients designated VV2. ${ }^{12}$ These findings suggest that some cortical areas are more severely affected than others in the same patient and that this distribution is influenced by the sCJD subtype. In our current study, we looked for differences in the MR lesion patterns among the $\mathrm{MM}, \mathrm{VV}$, and MV genotypes, but these differences did not reach statistical significance. This is probably due to the limited number of patients designated MV $(n=4)$ and VV $(n=$ 5) (On-line Table 3).

The normal variation of signal intensity in the cerebral cortex on FLAIR and DW imaging could also influence the detectability of the high cortical signal intensity. In healthy persons, DW imaging reveals signal hyperintensity predominantly near the skull base ${ }^{21,30}$ and on FLAIR in the amygdala, hippocampus, cingulate gyrus, and subcallosal area. ${ }^{21}$ These high-signal-intensity areas were explained for FLAIR by differences in the water content of cortical brain regions ${ }^{21}$ and by variations in T2 relaxation times, ${ }^{30}$ and for DW imaging by susceptibility artifacts. There is an overlap of normal cortical high signal intensity in healthy subjects and abnormally high signal intensity in patients with SCJD in certain brain regions such as the insula or cingulate gyrus, ${ }^{4,31}$ whereas there is no significantly higher signal intensity in patients with sCJD in the hippocampus or the temporobasal and frontobasal cortices, usually bright on FLAIR or DW imaging in healthy persons. ${ }^{21}$ We propose that the high conspicuity of cortical hyperintensity in areas such as the cingulate gyrus or the insula is the result of a combination of increased signal intensity due to underlying pathologic changes and the intrinsically higher signal intensity in these brain areas on DWI or FLAIR.

\section{Involvement of Deep Gray Matter}

Cortical hyperintensity was accompanied by high signal intensity in the basal ganglia and/or thalamus in 36 of 39 patients in the current study. This is more than reported by others. ${ }^{2,4,11}$ 
Because of the limited number of patients with cortical lesions without involvement of deep gray matter, comparing the distribution of the signal intensity changes among the groups did not reach statistical significance.

\section{Specificity of the Findings}

A limitation of our study was the lack of a control group. It would be interesting to compare our results with those from a group of patients with cortical signal intensity changes of other causes to test for specificity of the described pattern. Such a control group for sCJD should include the usual radiologic differential diagnoses of sCJD such as inflammatory, metabolic, and paraneoplastic brain diseases, hypoxia, postictal changes, brain tumors, and lymphoma. $3,11,32$

From a clinical standpoint, the most difficult differential diagnoses for sCJD are rapidly progressive Alzheimer disease, ${ }^{33-35}$ dementia with Lewy bodies, ${ }^{35}$ and multiple cerebral infarctions. ${ }^{36}$ In none of these diseases are cortical MR signal intensity changes usually present. Therefore, these diseases would not be part of such a comparison study.

\section{Conclusion}

There is a common MR pattern of cortical involvement in sCJD, which consists of high signal intensity in the insula, the cingulate and superior frontal gyri, and in the cortical areas near the midline. In patients with dementia who have the described cortical changes, the suspicion of sCJD should be raised even without involvement of the structures of the deep gray matter. The DW imaging allows easiest identification of the signal intensity changes and should therefore be included in the work-up of patients with suspected sCJD. The specificity of the described pattern needs to be evaluated further.

\section{Acknowledgments}

We thank all physicians who referred suspected patients to the German Reference Center for Spongiform Encephalopathies for providing clinical data, MRIs, and brain necropsies. We especially thank Hanno Schimikowski, Department of Radiology, University of Bonn, for his assistance with figure editing.

\section{References}

1. Human transmissible spongiform encephalopathies. Wkly Epidemiol Rec 1998;73:361-65

2. Schroter A, Zerr I, Henkel K, et al. Magnetic resonance imaging in the clinical diagnosis of Creutzfeldt-Jakob disease. Arch Neurol 2000;57:1751-57

3. Tschampa HJ, Kallenberg K, Urbach H, et al. MRI in the diagnosis of sporadic Creutzfeldt-Jakob disease: a study on inter-observer agreement. Brain 2005;128:2026-33

4. Young GS, Geschwind MD, Fischbein NJ, et al. Diffusion-weighted and fluidattenuated inversion recovery imaging in Creutzfeldt-Jakob disease: high sensitivity and specificity for diagnosis. AJNR Am J Neuroradiol 2005;26:1551-62

5. Tschampa HJ, Zerr I, Urbach H. Radiological assessment of Creutzfeldt-Jakob disease. Eur Radiol 2006 Nov 9; [Epub ahead of print]

6. Finkenstaedt M, Szudra A, Zerr I, et al. MR imaging of Creutzfeldt-Jakob disease. Radiology 1996;199:793-98

7. Collins SJ, Sanchez-Juan P, Masters CL, et al. Determinants of diagnostic investigation sensitivities across the clinical spectrum of sporadic CreutzfeldtJakob disease. Brain 2006;129:2278-87

8. Zeidler M, Collie DA, Macleod MA, et al. FLAIR MRI in sporadic CreutzfeldtJakob disease. Neurology 2001;56:282
9. Bahn MM, Parchi P. Abnormal diffusion-weighted magnetic resonance images in Creutzfeldt-Jakob disease. Arch Neurol 1999;56:577-83

10. Tschampa HJ, Murtz P, Flacke S, et al. Thalamic involvement in sporadic Creutzfeldt-Jakob disease: a diffusion-weighted MR imaging study. AJNR Am J Neuroradiol 2003;24:908-15

11. Shiga Y, Miyazawa K, Sato S, et al. Diffusion-weighted MRI abnormalities as an early diagnostic marker for Creutzfeldt-Jakob disease. Neurology 2004;63: 443-49

12. Parchi P, Giese A, Capellari S, et al. Classification of sporadic CreutzfeldtJakob disease based on molecular and phenotypic analysis of 300 subjects. Ann Neurol 1999;46:224-33

13. Zerr I, Schulz-Schaeffer WJ, Giese A, et al. Current clinical diagnosis in Creutzfeldt-Jakob disease: identification of uncommon variants. Ann Neurol 2000;48:323-29

14. Hamaguchi T, Kitamoto T, Sato T, et al. Clinical diagnosis of MM2-type sporadic Creutzfeldt-Jakob disease. Neurology 2005;64:643-48

15. Samman I, Schulz-Schaeffer WJ, Wohrle JC, et al. Clinical range and MRI in Creutzfeldt-Jakob disease with heterozygosity at codon 129 and prion protein type 2. J Neurol Neurosurg Psychiatry 1999;67:678-81

16. Fukushima R, Shiga Y, Nakamura M, et al. MRI characteristics of sporadic CJD with valine homozygosity at codon 129 of the prion protein gene and PrPSC type 2 in Japan. J Neurol Neurosurg Psychiatry 2004;75:485-87

17. Meissner B, Kortner K, Bartl M, et al. Sporadic Creutzfeldt-Jakob disease: magnetic resonance imaging and clinical findings. Neurology 2004;63:450-56

18. Collie DA, Summers DM, Sellar RJ, et al. Diagnosing variant Creutzfeldt-Jakob disease with the pulvinar sign: MR imaging findings in 86 neuropathologically confirmed cases. AJNR Am J Neuroradiol 2003;24:1560-69

19. Kretzschmar HA, Ironside JW, DeArmond SJ, et al. Diagnostic criteria for sporadic Creutzfeldt-Jakob disease. Arch Neurol 1996;53:913-20

20. Kretschmann H, Weinrich W. Cranial Neuroimaging and Clinical Neuroanatomy: Atlas of MIR Imaging and Computed Tomography, 3rd ed. Stuttgart, Germany: Thieme Medical Publishers; 2004

21. Hirai T, Korogi Y, Yoshizumi K, et al. Limbic lobe of the human brain: evaluation with turbo fluid-attenuated inversion-recovery MR imaging. Radiology 2000:215-470

22. Young GS, Lin Y, Chen N, et al. Abnormality of apparent diffusion coefficient (ADC) in somatosensory cortex in Jakob-Creutzfeldt disease (CJD): is rolandic cortex really spared? Paper presented at: Annual Meeting of the Radiological Society of North America; Nov 26-Dec 1, 2005; Chicago, Ill.

23. Matoba $\mathrm{M}$, Tonami $\mathrm{H}$, Miyaji $\mathrm{H}$, et al. Creutzfeldt-Jakob disease: serial changes on diffusion-weighted MRI. J Comput Assist Tomogr 2001;25:274-77

24. Rowley HA, McCue J, Salamat MS, et al. Diffusion and perfusion MRI in Creutzfeldt-Jakob disease. Proceedings of the International Society for Magnetic Resonance in Medicine 12th Scientific Meeting and Exposition. Berkeley, CA: International Society for Magnetic Resonance in Medicine; 2004:1340. Available from: http://cds.ismrm.org/ismrm-2004/Files/Program04.pdf

25. Urbach $\mathrm{H}$, Klisch J, Wolf HK, et al. MRI in sporadic Creutzfeldt-Jakob disease: correlation with clinical and neuropathological data. Neuroradiology 1998;40:65-70

26. Gertz HJ, Henkes H, Cervos-Navarro J. Creutzfeldt-Jakob disease: correlation of MRI and neuropathologic findings. Neurology 1988;38:1481-82

27. Mittal S, Farmer P, Kalina P, et al. Correlation of diffusion-weighted magnetic resonance imaging with neuropathology in Creutzfeldt-Jakob disease. Arch Neurol 2002;59:128-34

28. Kropp S, Schulz-Schaeffer WJ, Finkenstaedt M, et al. The Heidenhain variant of Creutzfeldt-Jakob disease. Arch Neurol 1999;56:55-61

29. Na DL, Suh CK, Choi SH, et al. Diffusion-weighted magnetic resonance imaging in probable Creutzfeldt-Jakob disease: a clinical-anatomic correlation. Arch Neurol 1999;56:951-57

30. Georgiades CS, Itoh R, Golay X, et al. MR imaging of the human brain at $1.5 \mathrm{~T}$ : regional variations in transverse relaxation rates in the cerebral cortex. AJNR Am J Neuroradiol 2001;22:1732-37

31. Tschampa HJ, Kallenberg K, Zerr I, et al. Pattern of cortical changes in sporadic Creutzfeldt-Jakob disease. Paper presented at: Annual Meeting of the Radiological Society of North America; Nov 26-Dec 1, 2005; Chicago, Ill.

32. Stadnik TW, Demaerel P, Luypaert RR, et al. Imaging tutorial: differential diagnosis of bright lesions on diffusion-weighted MR images [published erratum appears in Radiographics 2003;23:686]. Radiographics 2003;23:e7

33. Poser S, Mollenhauer B, Kraubeta A, et al. How to improve the clinical diagnosis of Creutzfeldt-Jakob disease. Brain 1999;122:2345-51

34. Zerr I, Pocchiari M, Collins S, et al. Analysis of EEG and CSF 14-3-3 proteins as aids to the diagnosis of Creutzfeldt-Jakob disease. Neurology 2000;55:811-15

35. Tschampa HJ, Neumann M, Zerr I, et al. Patients with Alzheimer's disease and dementia with Lewy bodies mistaken for Creutzfeldt-Jakob disease. J Neurol Neurosurg Psychiatry 2001;71:33-39

36. Steinhoff BJ, Zerr I, Glatting M, et al. Diagnostic value of periodic complexes in Creutzfeldt-Jakob disease. Ann Neurol 2004;56:702-08 